\title{
Computed Tomography Features and Clinicopathological Characteristics of Gastric Glomus Tumor
}

\section{Jingjing Xing}

Zhengzhou University First Affiliated Hospital

\section{Wenpeng Huang}

Zhengzhou University First Affiliated Hospital

\section{Fang Wang}

Zhengzhou University First Affiliated Hospital

\section{Yaru Chai}

Zhengzhou University First Affiliated Hospital

Jianbo Gao ( $\nabla$ jianboga063@163.com )

Zhengzhou University First Affiliated Hospital https://orcid.org/0000-0003-2621-3701

\section{Research Article}

Keywords: Glomus tumor, Gastric, Tomography, X-ray computed, Differential diagnosis

Posted Date: February 28th, 2022

DOI: https://doi.org/10.21203/rs.3.rs-877962/v1

License: (c) (1) This work is licensed under a Creative Commons Attribution 4.0 International License. Read Full License 


\section{Abstract \\ Background}

Gastric glomus tumor (GGT) is a rare neoplasm that lacks diagnostic experience, it is difficult to distinguish it from other gastric submucosal tumors. The objective of this study was to explore the clinicopathological features, computed tomography (CT) features and differential diagnosis of GGT to improve the understanding of the disease.

\section{Methods}

The clinical data and CT feature of 21 patients pathologically confirmed GGT were reviewed. The clinical data and CT features of benign GGT were compared with the gastric stromal tumors (GST) (n=30) and heterotopic pancreas $(n=30)$.

\section{Results}

21 cases included 6 males and 15 females, aged $42-64$ years. The lesion was in gastric body in 4 cases and gastric antrum in 17 cases. 20 cases of GGT were diagnosed as benign, 1 case was malignant. The glomus cells of benign cases were small, uniform, and round. Obvious mitotic figures and necrosis was observed in the malignant case. The tumor cells showed positive staining for SMA. 20 benign cases showed quasi-round or round solid mass protruded to the gastric cavity, the edge was clear and smooth. The ratio of long to short diameter was $1.01 \pm 0.15$. All benign cases showed obvious enhancement, including homogeneous enhancement in 10 cases and heterogenous enhancement in 10 cases, central filling enhancement was seen in 12 cases. The ratio of CT value of lesion to abdominal aorta in arterial phase and venous phase were $(0.41 \pm 0.11)$ and $(0.81 \pm 0.20)$, which were significantly higher than GST and heterotopic pancreas. In 1 malignant case, the long diameter was $150 \mathrm{~mm}$ and thick diameter was 30 $\mathrm{mm}$, the irregular mass broke through the gastric wall and invaded the liver with poorly defined boundary and internal necrosis, heterogeneous persistent moderate enhancement with thickening blood supply arteries was seen.

\section{Conclusions}

GGT is rare and most commonly occurs in the gastric antrum of middle-aged and elderly women. CT enhancement usually shows persistent obvious enhancement especially in arterial phase which provide important value for the diagnosis. CT findings can be contributed to the differential diagnosis of GGT and other submucosal tumors.

\section{Background}


Glomus tumors (GTs) are rare benign mesenchymal neoplasms originating from the glomus of the anastomoses of small arteries, usually occurring in the peripheral soft tissues, especially in the distal parts of fingertips or toes ${ }^{[1]}$. Glomus tumors rarely occur in the stomach ${ }^{[2]}$. GGT is mostly reported as a

case report ${ }^{[3-8]}$. As lack of diagnostic experience in imaging features, it is difficult to distinguish it from other gastric submucosal tumors perioperatively. In the present study, a total of 21 cases of GGTs proved by pathology were collected and analyzed. The clinicopathological features and CT findings were compared with those of gastric submucosal tumors such as gastric stromal tumor, heterotopic pancreas, expecting to improve the diagnosis of the GGT and narrow down the differential diagnosis.

\section{Materials And Methods}

\section{Patients}

This study is a retrospective study and has been approved by the Ethics Committee of the First Affiliated Hospital of Zhengzhou University, which waived the requirement of written informed consent because of anonymous information of the patients. Patients who met the following criteria were collected retrospectively in our hospital between January 2008 and June 2021. Inclusion criteria: $\otimes$ GGT confirmed by postoperative pathology; $₫$ Abdominal CT plain scan and contrast-enhanced scan were performed preoperatively. The clinical and pathological data were complete. Exclusion criteria: $\otimes$ There were obvious artifacts on the image; $₫$ The lesion was too small to be recognized. Clinical manifestations and tumor markers results were recorded for all patients.

\section{CT Evaluation}

Fifteen cases were examined with GE Discovery 750 HD CT, and 6 with Toshiba 320 CT. The scanning parameters for the former machine were: tube voltage of $120 \mathrm{kV}$, automatic $\mathrm{mA}$, pitch of 0.984 , thickness of $5 \mathrm{~mm}$; and the scanning parameters for the latter machine were: tube voltage of $120 \mathrm{kV}$, tube current of $350 \mathrm{~mA}$, pitch of 0.813 , thickness of $5 \mathrm{~mm}$, increment reconstruction. For each patient, contrast-enhanced images of the arterial phase and portal venous phase were acquired after an intravenous injection of nonionic iohexol (iopromide, $370 \mathrm{mg} / \mathrm{mL}$, GE Medical Systems, $1.5 \mathrm{~mL} / \mathrm{kg}$ and $3 \mathrm{~mL} / \mathrm{s}$ ) through a dualhead pump injector (Medrad, Warrendale, PA, United States). Contrast enhanced CT scans were obtained $25 \mathrm{~s}$ after administration of contrast agent for arterial phase and after 60 s for portal venous phase.

According to the gender, age ( \pm 3 years) and diagnosis year of each GGT patient matched with GST and heterotopic pancreas patients, $30 \mathrm{GST}$ and heterotopic pancreas patients were retrieved from picture archiving and communication system of our hospital. Two radiologists with 10 years and 30 years of experience interpreted the CT images independently. All images were uploaded to the GE ADW 4.6 station, and the coronal and sagittal images were reconstructed using the Multi-Planar Reformation(MPR) technique with slice thickness of $3 \mathrm{~mm}$ and slice spacing of $3 \mathrm{~mm}$. The degree of enhancement of the tumor was based on dynamic CT imaging using $\mathrm{HU}$ attenuation, where "obvious enhancement" was defined as $>40 \mathrm{HU}$, "moderate enhancement" as $>20 \mathrm{HU}$ and "mildly enhancement" as $<20 \mathrm{HU}$. 


\section{Results}

\section{Clinical manifestations}

Total of 21 GGT patients were included, including 6 males (28.6\%) and 15 females (71.4\%), with a male to female ratio of nearly $1: 2$, age range $42-64(54.70 \pm 7.27)$ years. 2 patients had a history of $H$. pylori infection. In the 20 benign cases, the chief complaint of 14 cases was abdominal pain, 2 cases had hematochezia, 2 cases had acid reflux and heartburn, 2 cases were found in physical examination. The median time from symptom onset to diagnosis was 9 months (3 to 60 months), and none of them had a history of digestive diseases or family genetic history. Tumor marker detection was not risen in the benign cases. In this group, 16 cases were treated surgically, 4 cases underwent gastroscopic resection. The comparison of clinical characteristics and CT features of glomus tumor, GIST, heterotopic pancreas is summarized in Table 1.

For the malignant case, a 63-year-old woman, the chief complaint was upper abdominal pain, the time from symptom onset to diagnosis was 4 months. Among tumor markers, CA-125 $(42.60 \mathrm{U} / \mathrm{ml})$ was increased. This case underwent distal gastrectomy and postoperative chemotherapy. There was no evidence of recurrence during the follow-up 16 months after the surgery.

\section{Pathological and other examinations}

Twenty cases of GGT were diagnosed as benign, only 1 case was malignant. Microscopically, tumor interspersed and grew between the muscularis propria of the gastric wall, thin-walled vessels were common, and small focal calcification was found in 1 case. The glomus cells were small, uniform, and round without nuclear pleomorphism or mitotic figures. 1 case was confirmed to be malignant, obvious mitotic figures and necrosis was observed, ulceration could be seen in the gastric mucosal surface (Fig 2E). In 21 cases of GGT, tumor thrombus was found in 4 cases, nerve invasion was found in 8 cases, involvement of submucosa and mucosa was found in 6 cases. On immunohistochemical examination, the tumor cells showed positive staining for Vimentin, SMA and Collagen-IV. CD34 was positive in 10 cases. Partial expression of Caldesmon or Calponin were moderately positive. Focal positivity for SYN was observed in 14 cases. The Ki- 67 index of the 20 benign cases were 1\% 5\%, the Ki-67 index of the malignant was $30 \%$. The results for the remaining stains, including CD56, CD117, CKPAN, DOG1, S-100 proteins were all negative in our group.

Twelve cases underwent endoscopic ultrasound, 6 cases were hypoechoic (Fig.1A), 4 cases were slightly hyperechoic, 2 cases were hyperechoic, echo of 6 cases were homogeneous and 6 cases were inhomogeneous. 6 cases received upper gastrointestinal radiography, 5 cases showed quasi-circular filling defect with smooth boundary (Fig.1B), and 1 case showed irregular filling defect and niche with obscure boundary (Fig.2A).

\section{CT findings}


All the 21 patients had single lesions. Among the 20 benign cases, 4 cases were in the gastric body, 16 cases were in the gastric antrum. 1 malignant case was located at the gastric antrum. 20 benign cases were quasi-round or round protruding into the gastric cavity with well-defined boundary, with no ulcers on the surface or signs of invasion (Fig 1C). Among the 20 benign GGTs, the ratio of long to short diameter was $1.01 \pm 0.15$, CT values of plain scan ranged from 27 to $54(38.60 \pm 8.21) \mathrm{Hu}$. All cases showed obvious enhancement in arterial phase, 10 cases showed homogeneous enhancement, 10 cases showed heterogeneous enhancement (Fig 1D), and central filling enhancement could be seen in 12 cases (Fig 1E,F). 12 patients showed persistent enhancement (the peak CT value occurred in venous phase), 6 patients showed reduced enhancement (the peak CT value occurred in arterial phase), and 2 patients showed continuous enhancement (CT value remained nearly the same toward arterial phase and venous phase). The ratio of CT value of lesion to abdominal aorta in arterial phase was $(0.41 \pm 0.11)$, and the ratio of $C T$ value of lesion to abdominal aorta in venous phase was (0.81 \pm 0.20$)$. In 1 malignant case, the long diameter was $150 \mathrm{~mm}$ and thick diameter was $30 \mathrm{~mm}$, the irregular mass broke through the gastric wall and invaded the liver with poorly defined boundary and internal necrosis (Fig 2D), heterogeneous persistent moderate enhancement was seen on the contrast CT images (Fig 2C,D), thickening left gastric artery supplying blood was seen in the arterial phase (Fig 2B).

\section{Discussion}

GT is rare in the stomach which accounts for approximately $1 \%$ of gastric mesenchymal tumors ${ }^{[9-10]}$ and was first reported by Kay et al in $1951^{[11]}$. GGT are commonly found in the gastric antrum. Among the 21 cases in this group, 17 cases occurred on gastric antrum, and 4 cases occurred on posterior wall of gastric body near gastric antrum, which is consistent with the literature report [12]. GGT is more common in middle-aged and elderly women with atypical clinical manifestations. As lack of typical clinical and endoscopic characteristics, GGT needs to be differentiated from other richly vascularized tumors such as GST and heterotopic pancreas ${ }^{[13]}$. Some patients may have gastrointestinal symptoms such as dull abdominal pain, acid regurgitation and belching, only a few patients present as gastrointestinal bleeding or chronic anemia. 15 cases were middle-old females in this group, the clinical symptoms of GGT were similar with other gastric submucosal tumors, showing no statistical difference of clinical symptoms compared with GST and heterotopic pancreas respectively. The vast majority of GGT are benign, malignant GGT are extremely rare ${ }^{[14]}$. In our group, 1 case presenting with upper abdominal pain was confirmed as malignant GGT. The etiology and mechanism of GGT are still not clear, while a few cases are familial and may be related to $1 \mathrm{p} 21-22^{[12]}$.

Glomus tumors are classified as vascular pericyte tumors in the WHO classification of soft tissue tumors. According to the different proportion of smooth muscle cells, globular cells and vascular components, GT can be divided into bulbar tumor, bulbar hemangioma, bulbar hemangiomyoma, and a few special types including myxoid hemangioma, eosinophilic hemangioma, epithelioid hemangioma, etc. However, tissue classification is not related to the biological behavior of the tumor. Folpe et al. divided GT into benign tumor, malignant tumor or tumor with uncertain malignant potential ${ }^{[15]}$. The histopathological 
characteristics of benign GT were obvious proliferation of smooth muscle cell and tumor nest surrounded by glomus cells around numerous small capillary vessels. The vast majority of glomus cells are small, round, and uniform without nuclear atypia or mitotic figures. The diagnosis of malignant GT includes three criteria: moderate to marked nuclear atypia together with mitotic activity of more than 5 mitoses per 50 high power fields, atypical mitotic figure or size of tumor more than $2 \mathrm{~cm}$ with deep location ${ }^{[16]}$. In our study, obvious mitotic figures, necrosis and ulceration of gastric mucosal surface were observed in 1 case which was confirmed to be malignant GGT. Immunohistochemical stains is positive for MSA, SMA, Collagen IV, H-caldesmon, Calponin and Vimentin, ant it is negative for CD-117, DOG1, desmin, chromogranin, S-100 protein and cytokeratin ${ }^{[17]}$.

GGTs are usually located in the submucosa and muscularis propria of the gastric wall. In our study, 12 cases underwent ultrasound gastroscopy showing low-echo or high-echo mass with homogeneous or inhomogeneous echo. Ultrasound gastroscopy can show the location of the tumor in the gastric wall, however, it is easily confused with other gastric submucosal tumors due to the lack of specificity. The upper gastrointestinal radiography showed round or quasi-circular filling defects with smooth boundary. Non-contrast CT images show single soft tissue lesion in the submucosa of gastric antrum with endophytic, exophytic or mixed growth. In our study, endophytic growth and mixed growth were more common. Most of GGTs are benign with smooth edge and well-defined boundary. Perigastric fat space is clear with no lymph node enlargement. Some literatures have reported spotty calcification in GGT due to the deposition of micro-phlebolith ${ }^{[18]}$. However, no fat or calcification was found on CT images of our group. CT can show smooth gastric mucosa covering the surface of the mass, suggesting that GGT originates from the submucosa. The enhanced degree was consistent with the surrounding gastric mucosa. No mucosal ulcer was observed in the 20 benign cases in this group, the volume was small, and the longest diameter of the lesions was all less than $3 \mathrm{~cm}$, which may be related to the slow growth of the tumor. Contrast enhanced CT images show obvious enhancement in arterial phase and persistent enhancement in venous phase. In our study, all the 20 benign cases showed obvious enhancement in arterial phase, central filling enhancement could be seen in 12 cases which was similar to the enhancement pattern of hemangioma corresponding to the rich capillary small vessels pathologically. 10 cases showed heterogeneous density on the contrast CT images, the area of low density was considered as cystic change caused by perivascular bleeding and degeneration ${ }^{[19]}$.

Malignant GGT is extremely rare which has been reported only in a few literature ${ }^{[14-16,20-21]}$. Most of these papers were case reports about pathology, however, no article about the radiological feature of malignant GGT was published to date. The imaging characteristics of malignant GGT need to be further studied. Malignant GGT can invade the whole gastric wall with poor-defined boundary. Toti $L$ et al. [14] reported one malignant GGT with liver metastases of a 72-year-old male patient with complaint of anemia, CT showed inhomogeneous mass which was $6 \mathrm{~cm}$ in diameter located in the greater curvature. There was no evidence of recurrence 3 and 6 months after the surgery. Alsahwan AG et al. ${ }^{[16]}$ reported one malignant GGT of s a-56-year-old male patient presented with upper gastrointestinal bleeding, CT images showed large lobulated mass at the greater curvature with no local invasion or distant 
metastasis. There was no evidence of recurrence 15 months after the resection of gastric mass. In our study, 1 case of malignant GGT mass showed deep ulcer with lobulation and nodular indentation on the surface, and the mass was large with exophytic growth invading the liver. The mass showed persistent moderate enhancement on contrast CT and the blood supply artery was from thickening left gastric artery. There was no evidence of recurrence 16 months after the surgery. Considering that few malignant glomus tumors have been reported at present, the CT findings of malignant glomus tumors need to be further studied and summarized.

CT examination can provide a wealth of information on the morphology, size, internal structure, growth pattern and blood supply of the lesion, and MPR can show the accurate location of the GGT. Due to the lack of typical clinical and endoscopic characteristics, GGT needs to be differentiated from other similarly manifesting gastric submucosal tumors such as GST and heterotopic pancreas ${ }^{[22,23]}$. GST usually occurs in the cardia and fundus of the stomach with exophytic growth ${ }^{[24]}$. In our study, GGT and GST showed significant differences in morphology, growth location, growth pattern, enhanced degree, enhanced pattern. GST was larger than $3 \mathrm{~cm}$ in size and showed persistent moderate enhancement on contrast CT images, and the ratio of CT value of lesion to abdominal aorta on contrast CT images was significantly lower than that of GGT. Heterotopic pancreas usually occurs in the gastric antrum, and most

of them are quasi-circular masses with obvious enhancement on contrast CT images ${ }^{[25-26]}$. In our study, GGT and heterotopic pancreas showed significant differences in morphology, growth location, growth pattern, enhanced degree, enhanced pattern, ratio of long to short diameter, ratio of CT value of lesion to abdominal aorta. There were no significant differences in the location or shape of GGT and heterotopic pancreas. Ratio of long to short diameter of heterotopic pancreas was higher than that of GGT. Because GGT is hypervascular tumor, it is not difficult to distinguish GGT from other submucosal tumors including gastric leiomyoma, gastric schwannoma, gastric neuroendocrine tumors. Gastric leiomyoma usually shows homogeneous density and mild to moderate enhancement. Gastric schwannoma usually combines with cystic degeneration and shows mild to moderate enhancement. The common CT manifestations of gastric neuroendocrine tumors (staged G1, G2) are mild to moderate enhancement on contrast CT.

\section{Conclusions}

GGT is rare and most commonly occurs in the gastric antrum of middle-aged and elderly women with atypical clinical manifestations, multiphase CT enhancement usually shows persistent obvious enhancement especially in arterial phase which provide important value for the diagnosis of GGT. The enhanced degree and pattern are based on its histological features. CT findings can be contributed to the differential diagnosis of GGTs and other gastric submucosal tumors. However, the final and correct diagnosis depend on the pathological and immunohistochemical results. It is necessary to continuously report and accumulate the newly diagnosed GGT, which can improve the clinical and imaging understanding of GGT. 


\section{Abbreviations}

CT

Computed tomography

GGT

Gastric glomus tumor

GT

Glomus tumor

GST

Gastric stromal tumor. MPR:Multi-Planar Reformation.

\section{Declarations}

\section{Ethics approval and consent to participate}

This study was approved by the Institutional Review Board of the First Affiliated Hospital of Zhengzhou University, and the requirement for informed consent was waived.

\section{Consent to publish}

Not applicable.

\section{Availability of data and materials}

The datasets used and/or analysed during the current study are available from the corresponding author on reasonable request.

\section{Competing interests}

The authors declare that they have no competing interest.

\section{Funding}

This work was supported in part by the National Natural Science Foundation of China, No. 81971615.

\section{Authors' Contributions}

JJX and WPH contributed equally to the manuscript. Study concept and design: JJX and WPH; Imaging data collection: $L M L$ and LYF; Imaging preliminary valuation: YRC and YC; Imaging final valuation: FW and JBG; Statistical analysis: LML and WPH; Manuscript preparation: JJX and WPH; All authors have read and approved the final manuscript.

\section{Acknowledgements}


Not applicable.

\section{Author details}

Department of Radiology, The First Affiliated Hospital of Zhengzhou

University, No.1, East Jianshe Road, Zhengzhou 450052, Henan Province.

\section{References}

1. Tsuneyoshi M, Enjoji M. Glomus tumor: a clinicopathologic and electron microscopic study. Cancer. 1982;50(8):1601-1607.

2. Miettinen M, Paal E, Lasota J, Sobin LH. Gastrointestinal glomus tumors: a clinicopathologic, immunohistochemical, and molecular genetic study of 32 cases. Am J Surg Pathol. 2002;26(3):301311.

3. Yoshida H, Asada M, Marusawa H. Gastrointestinal: Glomus tumor: A rare submucosal tumor of the stomach. J Gastroenterol Hepatol. 2019;34(5):815.

4. Devanathan M, Jamuna Rani S, Chandramohan SM, Anbarasu S. A rare submucosal tumour of stomach-glomus tumour: A case report. Int J Surg Case Rep. 2015;11:64-67.

5. Matevossian E, Brücher BL, Nährig J, Feußner H, Hüser N. Glomus tumor of the stomach simulating a gastrointestinal stromal tumor: a case report and review of literature. Case Rep Gastroenterol. 2008;2(1):1-5.

6. Chou HP, Tiu CM, Chen JD, Chou YH. Glomus tumor in the stomach. Abdom Imaging. 2010;35(4):390-392.

7. Cha SH, Cho SB, Kim YW, Park CM. Helical CT appearance of glomus tumor of the stomach. Eur Radiol. 2000;10(4):671-673.

8. Liu KL, Wang HP, Tseng WY, Shun CT, Chen SJ, Tsang YM. Glomus tumor of the stomach: MRI findings. AJR Am J Roentgenol. 2005;185(5):1190-1192.

9. Devanathan M, Jamuna Rani S, Chandramohan SM, Anbarasu S. A rare submucosal tumour of stomach-glomus tumour: A case report. Int J Surg Case Rep. 2015;11:64-67.

10. Castro Ruiz C, Carlinfante G, Zizzo M, Giunta A, Ronzoni R, Azzolini F, Pedrazzoli C. Glomus Tumor of the Stomach: GI Image. J Gastrointest Surg. 2017 Jun;21(6):1099-1101.

11. KAY S, CALLAHAN WP Jr, MURRAY MR, RANDALL HT, STOUT AP. Glomus tumors of the stomach. Cancer. 1951;4(4):726-736.

12. Gombos Z, Zhang PJ. Glomus tumor. Arch Pathol Lab Med. 2008;132(9):1448-1452.

13. Zhang S, Zhang J, Wang C. Glomus Tumor of the Stomach-A Tumor That Needs to Be Differentiated From Gastrointestinal Stromal Tumor. Clin Gastroenterol Hepatol. 2018;16(3):A29-A30. 
14. Toti L, Manzia TM, Roma S, Meucci R, Blasi F, Ferlosio A, et al. Rare malignant glomus tumor of the stomach with liver metastases. Radiol Case Rep. 2019;14(4):463-467.

15. Folpe AL, Fanburg-Smith JC, Miettinen M, Weiss SW. Atypical and malignant glomus tumors: analysis of 52 cases, with a proposal for the reclassification of glomus tumors. Am J Surg Pathol. 2001;25(1):1-12.

16. Alsahwan AG, Alfaraj ZM, AlSafwani J, Bunaiyan AH, AlKhalifah RH, Al-Saba'a SA,et al. Rare gastric neoplasm: Malignant glomus tumor of the stomach. A case report. Int J Surg Case Rep. 2021;81:105802.

17. Lin J, Shen J, Yue H, Li Q, Cheng Y, Zhou M. Gastric Glomus Tumor: A Clinicopathologic and Immunohistochemical Study of 21 Cases. Biomed Res Int. 2020;2020:5637893.

18. Hu SD, Hu DM, Huang W, Chen KM, Song Q. Computed tomography and clinical characteristics of gastric glomus tumors. J Dig Dis. 2014;15(9):477-482.

19. Kang G, Park HJ, Kim JY, Choi D, Min BH, Lee JH, et al. Glomus tumor of the stomach: a clinicopathologic analysis of 10 cases and review of the literature. Gut Liver 2012; 6: 52-7.

20. Teng TH, Huang SH, Liang CW. Malignant gastric glomus tumour mimicking GIST. Pathology 2012 Apr;44(3):261-263.

21. Miettinen M, Paal E, Lasota J, Sobin LH. Gastrointestinal glomus tumors: a clinicopathologic, immunohistochemical, and molecular genetic study of 32 cases. Am J Surg Pathol 2002 Mar;26(3):301-311.

22. Hur BY, Kim SH, Choi JY, Rha SE, Lee MW, Kim SY, et al. Gastroduodenal glomus tumors: differentiation from other subepithelial lesions based on dynamic contrast-enhanced CT findings. AJR Am J Roentgenol. 2011;197(6):1351-1359.

23. Baek YH, Choi SR, Lee BE, Kim GH. Gastric glomus tumor: Analysis of endosonographic characteristics and computed tomographic findings. Digestive Endoscopy. 2013;25(1):80-3.

24. Wang J, Liu C, Ao W, An Y, Zhang W, Niu Z, et al. Differentiation of gastric glomus tumor from small gastric stromal tumor by computed tomography. J Int Med Res. 2020;48(8):300060520936194.

25. Li LM, Feng LY, Chen XH, Liang P, Li J, Gao JB. Gastric heterotopic pancreas and stromal tumors smaller than $3 \mathrm{~cm}$ in diameter: clinical and computed tomography findings. Cancer Imaging. 2018 Aug 7;18(1):26.

26. Kim JY, Lee JM, Kim KW, Park HS, Choi JY, Kim SH, et al. Heterotopic pancreas: CT findings with emphasis on differentiation from small gastrointestinal stromal tumor and leiomyoma. Radiology. 2009;252(1):92-100.

\section{Tables}

Table 1 Comparison between GGT and GST, heterotopic pancreas 
GGT GST

heterotopic
pancreas $P_{1}{ }^{\mathrm{a}} \quad P_{2}{ }^{\mathrm{b}}$

Location

Antrum

Body

Cardia and Fundus

Main symptoms

Epigastric comfort/pain

Haematemesis / melena

Physical examination

Shape

Round

Quasi-round

Irregular shape

Growth Pattern

Endophytic

Exophytic

Mixed

Ratio of long to short

diameter

Enhancement degree

Mildly enhancement

Moderate enhancement

Obvious enhancement

Enhancement pattern

Persistent enhancement

Continuous enhancement

Reduced enhancement

Enhancement pattern

Homogeneous
$0.000 \quad 0.321$

0.721

0.119

\begin{tabular}{lll}
\hline $16(80.0 \%)$ & $21(70.0 \%)$ & $29(96.7 \%)$ \\
\hline $2(10.0 \%)$ & $5(16.7 \%)$ & $0(0.0 \%)$ \\
\hline $2(10.0 \%)$ & $4(13.3 \%)$ & $1(3.3 \%)$
\end{tabular}

$0.009 \quad 0.001$

\begin{tabular}{lll}
\hline $12(60.0 \%)$ & $12(40.0 \%)$ & $4(13.3 \%)$ \\
\hline $8(40.0 \%)$ & $7(23.3 \%)$ & $20(66.7 \%)$ \\
\hline $0(0.0 \%)$ & $11(36.7 \%)$ & $6(30.0 \%)$
\end{tabular}

$0.032 \quad 0.004$

$8(40.0 \%) \quad 23(76.7 \%) \quad 25(83.3 \%)$

$4(20.0 \%) \quad 2(6.7 \%) \quad 3(10.0 \%)$

$8(40.0 \%) \quad 5(16.7 \%) \quad 2(6.7 \%)$

$\begin{array}{lllll}1.01 \pm 0.15 & 1.18 \pm 0.30 & 1.61 \pm 0.84 & 0.02 & 0.003\end{array}$

\begin{tabular}{lll}
\hline $0(0.0 \%)$ & $4(13.3 \%)$ & $4(13.3 \%)$ \\
\hline $0(0.0 \%)$ & $16(53.3 \%)$ & $11(36.7 \%)$ \\
\hline $20(100.0 \%)$ & $10(30.3 \%)$ & $15(50.0 \%)$
\end{tabular}

$0.005 \quad 0.287$

$12(60.0 \%) \quad 24(80.0 \%) \quad 20(66.7 \%)$

$2(10.0 \%) \quad 6(20.0 \%) \quad 6(20.0 \%)$

$6(30.0 \%) \quad 0(0.0 \%) \quad 4(13.3 \%)$

$0.166 \quad 0.630$

$12(60.0 \%) \quad 12(40.0 \%) \quad 20(66.7 \%)$ 


\begin{tabular}{|c|c|c|c|c|c|}
\hline Heterogeneous & $8(40.0 \%)$ & $18(60.0 \%)$ & 10(33.3\%) & & \\
\hline $\begin{array}{l}\text { ratio of CT value of lesion to abdominal } \\
\text { aorta in arterial phase }\end{array}$ & $0.41 \pm 0.11$ & $0.20 \pm 0.05$ & $0.24 \pm 0.62$ & 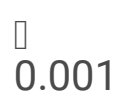 & $\begin{array}{l}\square \\
0.001\end{array}$ \\
\hline $\begin{array}{l}\text { ratio of CT value of lesion to abdominal } \\
\text { aorta in venous phase }\end{array}$ & $0.81 \pm 0.20$ & $0.48 \pm 0.12$ & $0.58 \pm 0.14$ & 0.001 & 0.001 \\
\hline
\end{tabular}

\section{Figure 1}

Images of a 50-year-old woman with benign GGT located on the greater curvature of gastric antrum. A. Endoscopic ultrasonography shows hypoechoic lesion with local heterogenous echo (white arrow), which originate from muscularis propria. B. Upper gastrointestinal barium meal radiography shows round filling defect with smooth boundary in the greater curvature of gastric antrum (red arrow). C. Non-contrasted CT shows round soft tissue density in the lesser curvature of the gastric antrum (arrowhead), the average CT value was about $41 \mathrm{Hu}$. D. Contrasted CT shows obvious enhancement in arterial phase, the average CT value was about $92 \mathrm{Hu}$. E. Portal venous phase image shows central filling enhancement, the average CT value was about $140 \mathrm{Hu}$. F. Portal phase of contrast enhancement coronal image shows homogenous density of the lesion with well-defined boundary.

\section{Figure 2}

Images of a 63-year-old woman with malignant GGT of gastric antrum. A. Upper gastrointestinal barium meal radiography shows a large filling defect in the lesser curvature of the stomach (red arrow). B. Arterial phase image shows irregular mass of gastric antrum with deeply lobulated margin, thickening left gastric artery supplying blood can be seen (white arrow). C. Portal venous phase image shows heterogeneous persistent moderate enhancement. D. coronal image of portal phase shows that the mass broke through the gastric wall and invade the liver with poorly defined boundary, cystic change and necrosis inside the tumor can be seen (arrowhead). E. Pathological images showed enlargement of nuclear volume, mitosis, and neoplastic necrosis in some areas (HE,100x). F. Tumor cells are positive for SMA upon immunohistochemical staining (100x). 